\title{
Capturing the dynamic and cyclical nature of regulation: Methodological Progress in understanding socially shared regulation in learning
}

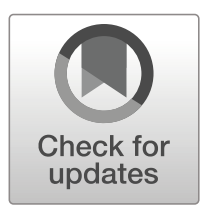

\author{
Sanna Järvelä ${ }^{1}$ (D) Hanna Järvenoja ${ }^{1} \cdot$ Jonna Malmberg $^{1}$
}

Received: 29 April 2019 / Accepted: 11 November 2019/Published online: 25 November 2019

(C) The Author(s) 2019

\begin{abstract}
Self-regulation is critical for successful learning, and socially shared regulation contributes to productive collaborative learning. The problem is that the psychological processes at the foundation of regulation are invisible and, thus, very challenging to understand, support, and influence. The aim of this paper is to review the progress in socially shared regulation research data collection methods for trying to understand the complex process of regulation in the social learning context, for example, collaborative learning and computer-supported collaborative learning. We highlight the importance of tracing the sequential and temporal characteristics of regulation in learning by focusing on data for individual- and group-level shared regulatory activities that use technological research tools and by gathering in-situ data about students' challenges that provoke regulation of learning. We explain how we understand regulation in a social context, argue why methodological progress is needed, and review the progress made in researching regulation of learning.
\end{abstract}

Keywords CSCL · Collaborative learning $\cdot$ Research methods · Self-regulated learning $\cdot$ Socially shared regulation

\section{Introduction}

Learning scientists want to understand how people learn. This compulsion is fueled by the wish to design, develop, and implement effective and efficient support and tools for learning success (Fischer et al. 2018). Unfortunately, this is challenging, as learning is a complex cognitive, metacognitive, motivational, and emotional process (Winne 2017). However, for decades, selfregulated learning (SRL) theory has helped us understand the key processes and features that

Sanna Järvelä

sanna.jarvela@oulu.fi

1 Department of Educational Sciences and Teacher Education, Learning and Educational Technology Research Unit (LET), University of Oulu, P.O. Box 2000, FIN-90014 Oulu, Finland 
promote or prevent learning. The ability and willingness to regulate one's learning is a critical skill for success in the twenty-first century, especially in terms of providing the means for an adaptive "life-long learning career" needed in the rapidly changing workplace (Luckin 2018), for moderating stress, and preventing school dropout (Salmela-Aro et al. 2017). Self-regulation, though, is a demanding skill that is best learned with the support of others and through access to selfregulation tools and/or environments (e.g., Hadwin et al. 2010).

While self-regulation of learning and working is difficult at the individual level, it is even more difficult when learning with others in groups where individual processes must be coordinated and the planning and execution thereof must be communicated between individuals (Kirschner et al. 2018). Collaborative learning and computer-supported collaborative learning (CSCL) produce more complex and multifaceted cognitive, motivational, and emotional challenges in learning than individual learning (Miyake and Kirschner 2014). Those challenges are the result of external (e.g., task difficulty), internal (e.g., weak strategies), and/or social (e.g., socioemotional conflicts) conditions (Järvenoja et al. 2013). Therefore, coregulation of learning (CoRL) and socially shared regulation of learning (SSRL) (Hadwin et al. 2018) are especially critical, as people need to continuously collaborate to solve today's and tomorrow's complex problems. Research has shown that groups do not recognize challenging learning situations and their need for regulation (Järvelä et al. 2016b), which restricts group members' activation of strategic adaptation in those situations (Rogat and AdamsWiggins 2015), and thus, they need to be alerted to this need.

Two decades of research have demonstrated that self-regulation is critical for successful learning (Dignath and Büttner 2008). Research on socially shared regulation has indicated that productive collaborative learning goes beyond individual group members' self-regulation and also includes sharing the responsibility of the regulation processes in addition to shared cognitive processing (Järvelä et al. 2016a). The challenge for the research is that the psychological processes at the foundation of regulation are multifaceted and, hence, very challenging to understand, support, and influence with current instrumentation. It is evident that reaching these foundational processes is dependent on methodological development (Cress et al. 2018). The aim of this paper is to review the progress in SSRL research data collection methods for trying to understand the complex process of regulation in the social learning context, for example, collaborative learning and CSCL. We explain how we understand regulation in a social context, argue why methodological progress is needed, and review the progress made in researching regulation of learning, especially in SSRL.

\section{SSRL helps understanding of complex learning process in collaboration}

Self-regulation refers to the ways that learners systematically activate and sustain their cognitions, motivations, behaviors, and emotions toward attaining their goals. Metacognitive monitoring, evaluation, and control fuel regulated learning (Schunk and Greene 2018). Therefore, we have adopted a multifaceted view of regulated learning, which means collecting data about the interplay among cognition, motivation, emotion, and metacognition during regulated learning and not just attending to a single facet.

Winne and Hadwin's $(1998,2008)$ model of individuals' SRL cycles is one of the many models explaining individuals' SRL and how self-regulation relates to motivation, emotions, and cognitive processing (see Schunk and Greene 2018). When SRL researchers became interested in extending self-regulation so as to consider regulation in collaborative learning settings, Winne and Hadwin's 
model became the premise for building theoretical and empirical understanding in group-level regulatory processes, namely socially shared regulation (Hadwin et al. 2018). They modeled regulation at a micro level with COPES (i.e., Conditions, Operations, Products, Evaluations, and Standards), which identifies facets of a task where learners exercise SRL. The COPES model describes learning as a cyclical process. When learners engage in SRL, they go through four phases, including developing task perception, goal setting and planning, translating plans into strategies according to the goals, and evaluation and reflection. The different phases of the cycle build upon each other in a loosely sequenced order and are cycled through COPES through metacognitive monitoring. As a result of the monitoring and consequent adapting of task perceptions, goals, and strategies, the sequencing enables shifting between the phases. Regulation of learning is active, particularly when learning is challenged or does not proceed according to the goals and standards (Järvelä et al. 2018).

Similar to individuals' activation of self-regulatory processes, regulation of learning that takes place within collaborative groups presumes students have a precise understanding and awareness of the sources of the emerging regulation needs, "what is going wrong", and of the targets for regulation. With strategic regulation, group members can influence various cognitive, motivational, and emotional processes taking place in the group and adjust their collaborative learning according to the task goals and shared standards. The group-level regulation is achieved through interaction among the grup members in parallel with the group members' self-regulatory cycles (Cleary and Zimmerman 2012; Sobocinski et al. 2017). Socially shared regulation, particularly, assumes reciprocity in regulatory actions between the group members. Self-regulation (regulating oneself), co-regulation (supporting each other), and socially shared regulation (regulating together) jointly form the relative regulated learning space in which individuals within the group regulate their own motivation, emotions, cognition, and behavior and simultaneously contribute to the groups' shared regulatory processes (Järvelä et al. 2018).

All these issues have consequences for methodological choices in the study of regulation in collaboration. When the aim is to understand how regulation "sets the stage" for collaboration, it cannot be observed solely at one point in time nor captured through learners' overall evaluations of their skills or retrospective appraisals of the regulation success. As the regulation space of a collaborative group is multifaceted, studying regulation calls for a multimethod approach, including both the process data sources capable of capturing regulation (inter)actions as well as the data regarding learners' individual characteristics and interpretations of the learning situation. In particular, the difficulties learners encounter offer a gateway to identifying the unfolding of regulation (Hadwin et al. 2018). For example, a researcher can identify how a certain moment of difficulty can inform the potential subsequent strategic regulation of learning by the student (Malmberg et al. 2015). Identifying segments of time when difficulties and strategic regulation of learning occur, in turn, creates opportunities for a researcher to examine how and when learners strategically adapt their cognition, motivation, or emotions.

\section{Current research challenges in understanding regulation in learning and collaboration}

We see currently three conceptual and related methodological research challenges in the field of SRL, especially regarding regulation in the social context. Each of these challenges needs to be met in order to move further in helping learners and groups better regulate their learning. 
The first challenge is that regulation is not linear; it involves cyclical adaptation, which is hard to capture with methodological means. It is not a state but rather a series of contingencies over time (Molenaar and Järvelä 2014), where learners use metacognitive monitoring and control to strategically adapt their learning if needed (Zimmerman 2013). Earlier approaches to SRL research, such as self-report instruments, were successful in identifying students' general beliefs about their learning (Pintrich 1995) as well as their tactics, strategies, and processes for achieving regulation in learning situations (Azevedo 2014). What has not been made clear is when those actions take place and how they influence each other, that is, examine the change in regulatory processes and types of regulation over time (temporality). Primarily, this gap has derived from the limited methodological ways of capturing this evolving process.

When self-regulation in learning involves multiple cycles of phases, such as planning and goal setting, enacting strategies, and evaluating the progress of learning through metacognitive monitoring (McCardle and Hadwin 2015), students' activation and success in regulation and progress in learning may vary across cycles (Sobocinski et al. 2017). Understanding the complexity of the phenomena and capturing cognitive, metacognitive, motivational, and emotional processes in the collaborative learning context requires tracing behavior over time (Ben-Eliyahu and Bernacki 2015). While most of the existing methods are able to accurately trace the learning events and students' regulatory acts (e.g., Bannert et al. 2014), they fail to provide indicators of why individual learners act in a particular way or how much individual learners in a group socially share these acts. Multiple data channels that target the same learning situations and events can provide and cross validate the events with other channels, such as video data, learning traces, and self-reports (Bernacki 2017). When considering collaborative learning and CSCL which follow the progress of collaboration, research should inform on the learning challenges that trigger learners' strategic adaptations of their task perceptions, goals, and strategies in different learning situations (Winne 2015). Moreover, the analysis methods should be able to capture the recursive bond from previous to forthcoming cycles.

The second challenge is to identify how three forms of regulation-SRL, CoRL, and SSRL (Hadwin) - together contribute to successful learning. The field has progressed past notions of learning as solely individual or solely collaborative. Research on SRL has made progress in anchoring research about regulated learning in specific psychological constructs, including (a) regulatory processes (i.e., monitoring, evaluating, and controlling) and (b) regulatory constructs or targets (i.e., motivation, cognition, behavior, and emotion). We have identified the critical processes of SSRL (Järvelä et al. 2015; Järvenoja et al. 2015) and have accumulated evidence about the contribution of SSRL to success in collaboration (Järvelä et al. 2016; Järvenoja et al. 2015; Zheng et al. 2019). Until now, research has focused mainly on the SSRL processes themselves (e.g., Lajoie et al. 2015; Rogat and Adams-Wiggins 2014; Schoor et al. 2015; Ucan and Webb 2015). Recent research findings, however, have strongly pointed out the importance of researching how these three forms of regulation (SRL, CoRL, and SSRL) together contribute to successful learning. Methodologically considering regulation of collaboration cannot be traced solely to interaction processes but presumes capturing individuals' subjective psychological premises and appraisals simultaneously.

The third challenge is that, in order to capture SSRL in the authentic learning context, student interactions within the social learning context need to be understood. SSRL conceptualizes regulation on a group level, and therefore, students' activities, choices, and outcomes are intertwined with dynamic internal, social, and environmental conditions, serving as affordances and constraints for regulation (Winne and Hadwin 2008). Collaborative learning 
conditions emphasize the situated and sociohistorical nature of regulation (Järvenoja et al. 2015) by recognizing features of the current situation, task, and contextual conditions (e.g., technological affordances); the knowledge and beliefs of the group (e.g., group dynamics); and a range of past experiences. Capturing the conditions for SSRL requires multiple data channels that target individual and social learning activities and their interactions with the learning context (e.g., available technology). The need for multiple layers of data that focus on both learners' individual metacognitive and shared social processes forces us to consider more data channels than we have had previously (D’Mello et al. 2017).

\section{Methodological progress in researching and understanding SSRL}

In light of the three abovementioned challenges, a need for ongoing methodological development is obvious. Given the cyclical and temporal nature of regulation in the social context, a need for new methods that can capture regulation in the (collaboration) process has been evident from the beginning (Schunk and Greene 2018). Emphasis in the field has been increasingly placed, for example, on real-time measurement that occurs as individuals are engaged in learning (Azevedo et al. 2017). This has been in an attempt to explain how selfregulation actually operates as learners engage with content; show how it changes over time and in response to changes in environmental conditions; and present it as a function of changes in learners' judgements, knowledge, and skills (Callan and Cleary 2017). When progressing our research in SSRL in collaborative learning, we have developed methods to (a) trace and understand the sequential and temporal characteristics of regulated learning, (b) focus on data regarding the individual- and group-level shared regulatory activities with technological tools, and (c) gather in-situ data about students' regulatory challenges in authentic learning task.

\section{Tracing sequential and temporal characteristics of regulated learning}

In light of the fact that regulation is not a static attribute over time within tasks and across tasks and situations (e.g., Zimmerman 2013) but, instead, is cyclically adaptive and, hence, fluctuating (identified first challenge from above), trace data can help in understanding the temporal progress of regulation (Molenaar and Järvelä 2014). For example, gStudy (Nesbit et al. 2006) is an advanced multimedia learning system that offers cognitive tools that students can use to work with course material but it also gives researchers an opportunity to study the SRL process. In gStudy, the students are provided with tools for making notes, tagging selected content, constructing new glossary entries, and drawing and manipulating concept maps to assemble information in order to process the information provided in the environment. We have been following Winne's (1982) idea of "trace" to describe data that reflect learners' engagements in learning tasks over time. Traces capture students' immediate learning activities without interrupting the learning process, thus making it possible to follow the students' use of different studying techniques in the learning context. Our contextual and event-based measures of regulated learning have followed microanalytic protocols (Cleary and Zimmerman 2001), targeted specific aspects of regulated learning, and focused on specific moments in time. Traces include time-stamped descriptions of observable interactions between learners and content, and gathered over time, they can track patterns and changes in regulatory processes. Research on the cognitive, motivational, emotional, and social aspects of learning (e.g., Malmberg et al. 2013, 2014) has shown that tracing learners' strategic activity as a continuous 
process and not just as a series of momentary snapshots can inform the quality of students' strategy use and, ultimately, their learning. For example, Malmberg et al. (2013) investigated in detail the differences between high- and low-achieving students' strategic actions in challenging and favorable learning situations when studying in a gStudy learning environment. The learning situation, whether challenging or favorable, was defined based on the students' situation-specific explanations before each gStudy learning session. Trace data gathered from these episodes were used to investigate the types of learning patterns that emerge among the high- and low-achieving students. The study evidenced that students' situation-specific interpretations did affect their SRL and strategy use depending on whether the situation was considered either challenging or favorable. In a similar way, Johnson et al. (2011) traced SRL by using think-aloud protocols during a problem-solving task. Their study revealed that learners use more planning processes later in the learning session and fewer learning strategies as their learning progresses. Both of the studies were able to capture learners' interpretations in a learning situation as well as the cycle of regulation processes.

The advantage of temporal and sequential analysis is that it can reveal how sequences of regulated learning unfold over time and can describe the associations between self-, co-, and socially shared regulated learning (Malmberg et al. 2017). The sequential characteristics of regulated learning show how the types and processes of regulated learning typically follow each other, while the temporal characteristics reveal when types and processes of regulated learning typically occur during the learning session or over time (Reimann et al. 2014). Azevedo (2014) argued that, if regulated learning is considered a cyclical feedback loop, it is important to understand how and why learners engage in various types and processes of regulated learning and, thus, what the key processes are that facilitate learning in regulated learning. Sobocinski et al. (2017) explored how groups of students changed their strategies between phases of regulated learning in different types of learning situations. To investigate changes between the phases, they used process discovery methods that are viable for illustrating how phases of regulation and transitions between these phases typically occur. Their study revealed that, when the groups perceived the learning situation as challenging, they switched between the planning and performance phases frequently. The results show that, during the challenging task, the groups needed to continuously adapt their planning and task understanding in order to accomplish the task during the performance phase. Therefore, the students' evolving understanding of the task affected the strategies they chose to use when performing the task.

The multimodal data can provide new supplementary and complementary ways for making visible and understanding important phases of regulated learning as they occur in challenging learning situations. Multimodal research data are data that originate from different data channels, such as self-reports, videos, eye tracking, or physiological data (Ochoa 2017). These methods can also significantly extend the current knowledge on the sequential and temporal nature of the complex learning processes (Azevedo et al. 2011). Similar to sequential fluctuation between the types of regulation, as was investigated in the above example, the socially shared regulation can be layered or sequenced according to the particular target of regulation, such as motivation, emotion, and cognition. Dindar et al. (2019), for example, studied high school students' $(N=31)$ advanced physics lessons where students worked on collaborative learning tasks in 15 sessions, and the researchers measured overall session-based physiological synchrony among the group members. They investigated the interplay of temporal changes in SRL processes (i.e., behavioral, cognitive, motivational, and emotional) and their relationship with learning achievement in collaborative learning. They found that physiological synchrony 
among the collaborating students was related only to cognitive regulation and that changes in motivational regulation were related to learning achievement. Pijeira-Díaz et al. (2019) worked with the same physiological data (electrodermal activity [EDA]), but correlated to arousal at the individual level, and they found that students collaborating in triads on a physics task were mostly at different arousal levels and that possible arousal contagion occurred mostly on a oneon-one basis within the triad. Earlier, they found that physiological coupling indices based on EDA provided an estimate of students' learning gain and the quality of their joint solution (Pijeira-Díaz et al. 2016).

\section{Focusing on data for individual- and group-level shared regulatory activities using technological research tools}

The second and third challenges address the fact that collaborative learning regulation is situated in an individual-social continuum rather than purely as an attempt to identify forms of regulation using technological tools.

Since earlier research has revealed that students do not always recognize the opportunities for regulation in collaboration (DiDonato 2013; Järvelä et al. 2013) or that the potential of CSCL and technological learning tools may be undermined by students' inability or unwillingness to regulate learning (Azevedo 2005; Shapiro and Niederhauser 2004), researchers have developed technological tools to prompt and support self-regulation (Azevedo and Witherspoon 2009; Miller and Hadwin 2015), co-regulation, and socially shared regulation (Järvelä et al. 2016). Most tools have been found to support individual SRL and metacognitive monitoring (Bannert and Reimann 2012; Schnaubert and Bodemer 2017), while less efforts have been made to support socially shared regulation in groups. However, there is a potential to focus on data for individual- and group-level shared regulatory activities using technological research tools.

Our current work has concentrated on, for example, tracing how individuals in a group engage in planning and reflection by using tools for prompting a) individual and group planning and reflection processes (Malmberg et al. 2015); b) collective visualizations of individual plans and perceived challenges (Miller and Hadwin 2015); and c) emotional, cognitive, and motivational states (Järvelä et al. 2016). All of these tools have also been used for data collection as well as to identify how shared regulation is actualized when they are prompted. The first guiding principle in development of these tools has been to support regulation by prompting learners and groups to increase awareness of their own, others', and their group's appraisals of the conditions for and challenges with the learning processes to make these features of the learning process visible on the social plane. Another principle has been to make the individual and socially shared regulation visible for the group members and, thus, increase SSRL. Awareness of the situational features and accurate recognition of the requirements of the situation (the first principle) are conditions for these timely and accurate adaptations of regulatory actions. They serve as conditions for the second design principle (see Järvelä et al. 2015).

Methodologically, these support tools and prompts have offered a new way to record data "on the fly" that are not available by other means. For example, in MetaTutor (Azevedo 2015), learners are given prompts that promote metacognitive awareness in each phase of the regulated learning cycle. Johnson et al. (2011) detected, traced, modeled, and fostered students' self-regulatory and metacognitive processes during learning in hypermedia learning environments and identified the critical monitoring processes that triggered regulated learning. 
Technological advancement in research on SRL is the increasing use of technology for supporting learning and interaction processes as well as for data collection. CSCL provides a new source of data for tracing learning processes, and new data-driven analytical techniques are available (Reimann et al. 2014). Tools for the regulation process utilize, for example, software features to ease learners' navigation in a learning environment (Puntambekar and Stylianou 2005), to assist information processing by offering cognitive scaffolds for learning strategy use (Malmberg et al. 2010; Perry et al. 2010), to provide visualizations that track the progress of the learning process (Narciss et al. 2007), or to increase awareness of the collaborative learning process with mirroring tools (Fransen et al. 2011).

The difficulty is that learners do not necessarily externalize the thoughts that might signal their attempts to self-regulate their learning. Miller and Hadwin (2015) suggested that regulation of learning can be made visible using scripts and awareness tools that model SRL and report on the SRL process. Bakhtiar et al. (2018) developed a narrative constructor that prompts learners to identify a main challenge and a strategy from a dropdown list to overcome it. The narrative constructor tool includes a Socio-Emotional Sampling Tool (SEST) for the planning phase (Webster and Hadwin 2014) that prompts students to identify their feelings in the present moment and a strategy they could use to regulate that feeling. Students have access to their responses on the SEST when reflecting upon and describing their emotion regulation strategies in the individual reflection. The advantage of a narrative constructor is that it intuitive to using meaning and that it can capture events in a learning situation without interrupting the learning process (Aleven et al. 2010). Moreover, it provides valuable information for students and researchers about mental processes that either hamper or promote collaborative learning.

Our aim has been to capture individual SRL activities as a part of SSRL. For that, we have tailored and modified technological tools to prompt SSRL and combine the individual and group levels. These include structured tools that prompt students to negotiate and share their goals, plans, and strategies as well as offer the possibility of reflecting on whether the goals were achieved, whether the plans were adequate, and how effective the strategies were (Järvelä et al. 2015). The S-Reg tool was developed to prompt a group's awareness of their motivation, emotion, and cognitive conditions for collaboration in situ (Järvelä et al. 2016). With the S-Reg tool, students were prompted to anticipate the challenges and discuss and adapt their regulation processes upfront according to the situational conditions. The S-Reg tool increased coregulation, which may indicate that it supported awareness of individual students' situational motivations and emotions and became concrete in shifting support with a peer (Järvenoja et al. 2017). As co-regulation was increased, particularly at the beginning of the collaborative learning session, it created a condition for collaborative learning for the latter phases because it tapped the unfavorable aspects in the beginning (Kimmel and Volet 2010; Zschocke et al. 2016).

\section{Gathering in-situ data about students' challenges provoking regulation in authentic learning tasks}

Regulation situations arise over the course of learners' actions within a given learning context, as identified in the third challenge. Those situations are formed and shaped via interaction between learners and contextual features (Järvelä et al. 2010). Therefore, regulation activities during cyclical adaptation of the regulated learning are not independent from each other but are embedded in the timeframes in specific learning contexts, and thus, all regulatory activities, 
whether past or present, serve to learn from self-regulation (Cress and Hesse 2013; Molenaar and Chiu 2014). Hadwin et al. (2011) proposed that challenge episodes can be considered triggers that potentially activate regulatory activities; challenges are critical indicators for tracking and sequencing process data in order to locate the regulation taking place to overcome these challenges. Through the experienced and observable challenges and learners' subjective appraisals of them, the researchers can understand the reason for regulation. These reasons are also contextualized and formed as a part of the cyclical process involving learners' interactions with the task, contexts, social interactions, beliefs, technological tools, and artefacts.

Analyzing regulation within challenge episodes sharpens the focus on tracking separate regulatory activities over the course of collaboration. Koivuniemi et al. (2017) studied higher education students and found that they experienced a wide range of challenges regarding their cognitive and motivational processes as well as their emotions and well-being in collaborative learning tasks. Recently, researchers have used different classification schemes for analyzing the types of challenges and related regulation. Some of the studies have focused on identifying a certain type of challenge, such as cognitive, motivational, or socioemotional, that occurs during collaborative learning (Koivuniemi et al. 2017; Summers and Volet 2010). Other studies have recognized and categorized challenges and depicted the nature of regulation that the groups activate in relation to the different challenges (Malmberg et al. 2015). Yet, a pitfall of the abovementioned studies is that challenges are not recognized a priori but rather a posteriori of when they actually occur. Analytical progress is especially needed to unpack the contextualized set of factors that together compose the reason challenges are experienced in situ, thus influencing how regulation is adapted.

We have gathered in-situ data about students' challenges and related regulation in authentic learning tasks. This is to capture the full complexity of the regulation in learning situations involving learners' interactions with the task, context, social interaction, beliefs, technological tools, and artefacts. We have built on a situative perspective that allows us to consider the unique constitution of individual and social features of situated challenges and to follow the regulation as it evolves within a given situation (Järvenoja et al. 2015). The students' personal experiences, group dynamics, and task characteristics have all been found to influence the cognitive (e.g., challenges in terms of understanding a task), motivational (e.g., task commitment problems), and emotional (e.g., overruling interaction) challenges emerging within collaborative groups. That is, the context influences the nature of the regulation (e.g., whether regulation strategies seek to overcome a challenge or to avoid it) (Järvenoja et al. 2015; Volet and Järvelä 2001). Methodologically speaking, this is to combine different data sources or analyses to understand the unfolding regulation processes and/or the interplay between individual- and group-level regulation processes.

For example, Järvenoja et al. (2018) studied emerging challenges and related emotion regulation during teacher education students' collaborative work across six different mathematics tasks. The analysis combined a series of video data coding tiers, (i.e., challenges, type of regulation, and regulation strategy) to investigate the complex time-related connections and the fluctuation between the situation-specific challenges and regulatory activity in the group. The analysis revealed that, in collaborative learning situations, a wide range of micro-level challenges emerge, including those with cognitive, motivational, and emotional issues as well as different socially and contextually oriented challenges. All of these challenging situations are possible triggers for group-level regulation to occur. The strength of this type of analysis is that it is able to cross the boundary between motivational, emotional, and cognitive challenges because the analysis is based on the actualized behavior in situ. Moreover, interconnecting 
different actualized components of the regulation activity (i.e., challenge), type of regulation, and strategy, it can rise above the situatedness to reveal the typical patterns in the chained challenge-regulation-strategy behavior while still acknowledging their contextualized nature. In addition, Hadwin et al. (2018) investigated how specific support in the form of visualizations given for collaborating groups at the planning phase affects their regulation and learning outcomes. The groups who received visualization support at the planning phase reported effectiveness in terms of planning and teamwork strategies, and these groups were more successful in their strategy than groups without visualization support. An essential feature of this visualized support was that it was connected to a certain context and learning task; namely, it was situated with several current contextual features the students were connected to (i.e., task, group, and personal appraisals).

The situative perspective (Greeno 1998) allows for multilayered consideration of the collaborative groups' regulation activities in challenging situations and provides methodological solutions for capturing the complex relationships between different context-bound factors (Cress and Hesse 2013; Wise and Chiu 2011). Several process-oriented methods (Volet and Vauras 2013) and microanalytical protocols (Cleary et al. 2012) have been developed to identify traces of regulation as temporally unfolding events that are contextualized in situ. A fine-grained form of microanalytical protocols that targets behaviors or processes as they occur in real time across multiple learning contexts, and hence, is able to trace generalizable processes without losing the specific contextualized features, has yet to be developed. Until now, the majority of existing studies have used microanalysis to directly observe overt microlevel behaviors during interactions (Bannert et al. 2014). As these protocols are increasingly able to acknowledge covert processes, the possibility of capturing the process of regulated learning in real time in various learning contexts and with technological tools continues to evolve (Azevedo and Aleven 2013).

\section{Conclusion and discussion}

In this paper, we have discussed the progress in SSRL research data collection methods for understanding regulation in collaborative learning and CSCL. Methodological advancement in SSRL can help in the understanding of the complex process of collaboration and further practical implications in CSCL. CSCL research data comprise collaborative and socioemotional interactions, transactions, and knowledge-building processes. They have a temporal (in terms of time), multidimensional (in terms of affect and cognition), and cyclical nature (in terms of interacting and contributing to each process) that, in the case of good collaboration, are successfully shared among the collaborating partners (Järvelä and Hadwin 2013).

The methodological progress and the type of available data that have followed this progress have already contributed to the conceptual progress and understanding of the mechanisms in $\mathrm{S}(\mathrm{SRL})$. We have been successful in identifying relevant SRL traces from log data (Bannert et al. 2015; Malmberg et al. 2014). In addition, we have used advanced data-mining techniques to examine sequential patterns in regulation over time among high- and low-achieving students (Malmberg et al. 2017; Molenaar and Chiu 2014) to describe the learning activities in which a student typically is involved and to inform the actual process of SRL and SSRL (Järvelä et al. 2013; Johnson et al. 2011; Zheng et al. 2019). Progress has also been made in adequately researching regulation data sampled over time, both within and across episodes (Järvelä et al. 2016a; Paans et al. 2018). To generate methods for measuring regulated learning "on the fly" 
and in actual actions in a social learning context, we have studied learning in authentic collaborative groups and integrated situated pedagogical designs and data collection tools as well as support for SSRL (see Järvelä et al. 2015, 2016b).

The methodological progress for the field is dependent on how successfully we will be able to bring in more markers for understanding the complex process of regulation of cognition, motivation, and emotion, and how they are developed by widening data channels in terms of multimodal data. It is critical that empirical studies address the complex process of the analyses to avoid fragmentation. In this regard, multimodal approaches in SRL research can help tackle the constraints of typical single-channel data and help draw more valid and reliable inferences about the learning processes (Harley et al. 2015). While subjective measures (e.g., self-report data and interviews) explore the intent and appraisal of student learning, objective data (e.g., log data, eye tracking, heart rate, electro-dermal activity, videos, and facial expressions) can provide "on-the-fly" information about what students do when studying and can detect phases of challenge, interest, and attention (Henriques et al. 2013). Although interest in applying multimodal data has increased in SRL, SSRL, and CSCL research, it is still in the early stages. This is mostly because multimodal data come with different sampling rates, and depending on the type of data, there might be difficulties aligning the multimodal data. Also, more evidence is needed as to exactly what the different modalities (e.g., electrodermal activity, heart rate) reveal about the learning and collaboration processes.

The challenge still remains that there are no "metrics" for measuring mechanisms of regulation in learning in general and SSRL specifically. In stable conditions, "flat explanations," such as patterns, models, and regularities, can be predictive (Reimann et al. 2014), but these conditions are seldom the case in complex social situations. Therefore, data's correspondence to observed events are issues for consideration in future studies (Winne 2019), and more work is needed to integrate the strong tradition of microanalytical methods for studying collaboration, such as conversation and interaction analysis (e.g. Lund and Suthers 2018), into the new methodological approaches. This, in turn, would be detrimental for developing the "next generation" of support that would be more precise and targeted by the contextual features as well as temporally accurate and well situated in the regulation cycle.

Another major future challenge is to progress from the analyses of the temporal process of regulation in collaboration as small-scale regulatory acts to large-scale adaptations and their contribution to the progress of collaborative learning and individual long-term learning. Larger multimodal data sets in which the data are layered not only by situated modalities but also in time will open avenues for investigating the effects of adaptive regulation on individual development. Interdisciplinary collaboration in learning process analytics (e.g. Knight et al. 2017) will bring the next wave of research in SSRL and CSCL by especially widening the time scale from analyzing collaborate tasks to several recursive sessions of many collaborative groups.

Finally, concerning CL and CSCL, Kirschner et al. (2018) discussed how transactive activities play a crucial role in collaborative learning and pointed out that learning is particularly likely to occur when the collaborating students engage in transactive discourse (i.e., critique, challenging of positions, and attainment of synthesis via discussion), as this form of discourse gives rise to cognitive activities that stimulate knowledge construction. Clearly, collaborative learning effort is influenced by how well students coordinate their activities across time and transact with each other's ideas. A concept of socially shared regulation in learning adds to the understanding of what "sharing" cognition, motivation, and emotion among members in collaborative learning groups entails. Collaboration requires negotiating beliefs and perceptions regarding the collaborative goals and plans of how to achieve the task. 
This is a complex process for the co-construction of goals and plans, where metacognition and regulation processes of collaborating individuals need to be exchanged, negotiated, and aligned to achieve shared or joint regulation (Järvelä et al. 2018).

Conceptual and methodological progress made in research on SSRL will advance research on CSCL and can provide answers to the question "How do learners build a shared understanding of the task in a group and strategically regulate their collaborative learning process for a joint task to be achieved?" Since the main interest in CSCL supports the fine-grained processes that make collaboration and joint learning activities happen (Cress et al. 2018), one can conclude that many of the problems encountered when using CSCL in practice might be solved if we had tools at our disposal that could help the participants in CSCL groups regulate learning within the groups. In the future, technology can play a major role in both helping researchers understand the complex processes behind collaboration and helping regulate learning and support groups for more efficient collaboration.

Acknowledgements This study was supported by the Finnish Academy grants 275440; 308809; 297686. Oulu University LeaF research infrastructure has been used in data collection.

Funding information Open access funding provided by University of Oulu including Oulu University Hospital.

Open Access This article is distributed under the terms of the Creative Commons Attribution 4.0 International License (http://creativecommons.org/licenses/by/4.0/), which permits unrestricted use, distribution, and reproduction in any medium, provided you give appropriate credit to the original author(s) and the source, provide a link to the Creative Commons license, and indicate if changes were made.

\section{References}

Aleven, V., Roll, I., McLaren, B. M., \& Koedinger, K. R. (2010). Automated, unobtrusive, action-by-action assessment of self-regulation during learning with an intelligent tutoring system. Educational Psychologist, 45(4), 224-233. https://doi.org/10.1080/00461520.2010.517740.

Azevedo, R. (2005). Using hypermedia as a metacognitive tool for enhancing student learning? The role of selfregulated learning. Educational Psychologist, 40(4), 199-209. https://doi.org/10.1207/s15326985ep4004_2.

Azevedo, R. (2014). Issues in dealing with sequential and temporal characteristics of self- and socially-regulated learning. Metacognition and Learning, 9(2), 217-228. https://doi.org/10.1007/s11409-014-9123-1.

Azevedo, R. (2015). Defining and measuring engagement and learning in science: Conceptual, theoretical, methodological, and analytical issues. Educational Psychologist, 50(1), 84-94. https://doi.org/10.1080 /00461520.2015.1004069.

Azevedo, R., \& Aleven, V. (2013). Metacognition and Learning Technologies: An Overview of Current Interdisciplinary Research. In R. Azevedo \& V. Aleven (Eds.), International Handbook on Metacognition and Learning Technologies (pp. 1-16). New York: Springer. https://doi.org/10.1007/978-1-4419-5546-3_1.

Azevedo, R., \& Witherspoon, A. M. (2009). Self-regulated learning with hypermedia. In D. J. Hacker, J. Dunlosky, \& A. C. Graesser (Eds.), Handbook of metacognition in education (pp. 319-339). New York: Routledge.

Azevedo, R., Cromley, J. G., Moos, D. C., Greene, J. A., \& Winters, F. I. (2011). Adaptive content and process scaffolding: A key to facilitating students' self-regulated learning with hypermedia. Psychological Test and Assessment Modeling, 53(1), 106-140.

Azevedo, R., Taub, M., \& Mudrick, N. V. (2017). Understanding and reasoning about real-time Cognitive, Affective, and Metacognitive Processes to Foster Self-Regulation with Advanced Learning Technologies. In D. Schunk \& J. A. Greene (Eds.), Handbook of Self-Regulation of Learning and Performance (pp. 275292). London: Routledge. https://doi.org/10.4324/9781315697048.ch17.

Bakhtiar, A., Webster, E. A., \& Hadwin, A. F. (2018). Regulation and socio-emotional interactions in a positive and a negative group climate. Metacognition and Learning, 13(1), 57-90. https://doi.org/10.1007/s11409017-9178-x. 
Bannert, M., \& Reimann, P. (2012). Supporting self-regulated hypermedia learning through prompts. Instructional Science, 40(1), 193-211. https://doi.org/10.1007/s11251-011-9167-4.

Bannert, M., Reimann, P., \& Sonnenberg, C. (2014). Process mining techniques for analysing patterns and strategies in students' self-regulated learning. Metacognition and Learning, 9(2), 161-185. https://doi. org/10.1007/s11409-013-9107-6.

Bannert, M., Sonnenberg, C., Mengelkamp, C., \& Pieger, E. (2015). Short- and long-term effects of students' self-directed metacognitive prompts on navigation behavior and learning performance. Computers in Human Behavior, 52, 293-306. https://doi.org/10.1016/j.chb.2015.05.038.

Ben-Eliyahu, A., \& Bernacki, M. L. (2015). Addressing complexities in self-regulated learning: a focus on contextual factors, contingencies, and dynamic relations. Metacognition and Learning, 10(1), 1-13. https://doi.org/10.1007/s11409-015-9134-6.

Bernacki, M. L. (2017). Examining the cyclical, loosely sequenced, and contingent features of self-regulated learning: trace data and their analysis. In D. H. Schunk \& J. A. Greene (Eds.), Handbook of self-regulation of learning and performance (pp. 395-412). London: Routledge.

Callan, G. L., \& Cleary, T. J. (2017). Multidimensional assessment of self-regulated learning with middle school math students. School Psychology Quarterly, 33(1), 103-111. https://doi.org/10.1037/spq0000198.

Cleary, T. J., \& Zimmerman, B. J. (2001). Self-Regulation Differences during Athletic Practice by Experts, NonExperts, and Novices. Journal of Applied Sport Psychology, 13(2), 185-206. https://doi.org/10.1080 /104132001753149883.

Cleary, T. J., \& Zimmerman, B. J. (2012). A cyclical self-regulatory account of student engagement: Theoretical foundations and applications. In S. L. Christenson, A. L. Reschly, \& C. Wylie (Eds.), Handbook of research on student engagement (pp. 237-257). Boston: Springer. https://doi.org/10.1007/978-1-4614-2018-7_11.

Cleary, T. J., Callan, G. L., \& Zimmerman, B. J. (2012). Assessing Self-Regulation as a Cyclical, ContextSpecific Phenomenon: Overview and Analysis of SRL Microanalytic Protocols. Education Research International, 2012, 1-19. https://doi.org/10.1155/2012/428639.

Cress, U., \& Hesse, F. W. (2013). Quantitative Methods for Studying Small Groups. In C. E. Hmelo-Silver, C. A. Chinn, C. K. K. Chan, \& A. O’Donnell (Eds.), The International Handbook of Collaborative Learning (pp. 85-111). New York: Routledge. Taylor \& Francis. https://doi.org/10.4324/9780203837290.ch5.

Cress, U., Stahl, G., Rose, C., Law, N., \& Ludvigsen, S. (2018). Forming social systems by coupling minds at different levels of cognition: Design, tools, and research methods. International Journal of ComputerSupported Collaborative Learning, 13(3), 235-240. https://doi.org/10.1007/s11412-018-9284-z.

D’Mello, S., Dieterle, E., \& Duckworth, A. (2017). Advanced, Analytic, Automated (AAA) Measurement of Engagement During Learning. Educational Psychologist, 52(2), 104-123. https://doi.org/10.1080 /00461520.2017.1281747.

DiDonato, N. C. (2013). Effective self-and co-regulation in collaborative learning groups: An analysis of how students regulate problem solving of authentic interdisciplinary tasks. Instructional Science, 41(1), 25-47. https://doi.org/10.1007/s11251-012-9206-9.

Dignath, C., \& Büttner, G. (2008). Components of fostering self-regulated learning among students. A metaanalysis on intervention studies at primary and secondary school level. Metacognition and Learning, 3(3), 231-264. https://doi.org/10.1007/s11409-008-9029-x.

Dindar, M. Malmberg, J., Järvelä, S., Haataja, E. , \& Kirschner, P. A. (2019). Matching self-reports with electrodermal activity data: Investigating temporal changes in self-regulated learning. Education and Information Technologies.

Fischer, F., Hmelo-Silver, C. E., Goldman, S. R., \& Reimann, P. (2018) (Eds.). International Handbook of the Learning Sciences. New York: Routledge. Taylor \& Francis.

Fransen, J., Kirschner, P. A., \& Erkens, G. (2011). Mediating team effectiveness in the context of collaborative learning: The importance of team and task awareness. Computers in Human Behavior, 27(3), 1103-1113. https://doi.org/10.1016/j.chb.2010.05.017.

Greeno, J. G. (1998). The situativity of knowing, learning, and research. The American Psychologist, 53(1), 526. https://doi.org/10.1037/0003-066X.53.1.5.

Hadwin, A. F., Oshige, M., Gress, C., \& Winne, P. (2010). Innovative ways for using gStudy to orchestrate and research social aspects of self-regulated learning. Computers in Human Behavior, 26, 794-805. https://oi. org/10.1016/j.chb.2007.06.007.

Hadwin, A., Järvelä, S., \& Miller, M. (2011). Self-Regulated, Co-Regulated, and Socially shared Regulation of Learning. In B. Zimmerman \& D. Schunk (Eds.), Handbook of Self-Regulation of Learning and Performance (pp. 65-84). New York: Routledge.

Hadwin, A. F., Bakhtiar, A., \& Miller, M. (2018). Challenges in online collaboration: Effects of scripting shared task perceptions. International Journal of Computer-Supported Collaborative Learning, 13(3), 301-329. https://doi.org/10.1007/s11412-018-9279-9. 
Harley, J. M., Bouchet, F., Hussain, M. S., Azevedo, R., \& Calvo, R. (2015). A multi-componential analysis of emotions during complex learning with an intelligent multi-agent system. Computers in Human Behavior, 48, 615-625. https://doi.org/10.1016/j.chb.2015.02.013.

Henriques, R., Paiva, A., \& Antunes, C. (2013). On the need of new methods to mine electrodermal activity in emotion-centered studies. In L. Cao, Y. Zeng, A. L. Symeonidis, V. I. Gorodetsky, P. S. Yu, \& M. P. Singh (Eds.), Agents and Data Mining Interaction (Vol. 7607, pp. 203-215). Berlin: Springer. https://doi. org/10.1007/978-3-642-36288-0 18.

Järvelä, S., \& Hadwin, A. (2013). New Frontiers: Regulating learning in CSCL. Educational Psychologist, 48(1), 25-39. https://doi.org/10.1080/00461520.2012.74800.

Järvelä, S., Volet, S., \& Järvenoja, H. (2010). Research on motivation in collaborative learning: Moving beyond the cognitive-situative divide and combining individual and social processes. Educational Psychologist, 45(1), 15-27. https://doi.org/10.1080/00461520903433539.

Järvelä, S., Järvenoja, H., Malmberg, J., \& Hadwin, A. F. (2013). Exploring socially shared regulation in the context of collaboration. Journal of Cognitive Education and Psychology, 12(3), 267-286. https://doi. org/10.1891/1945-8959.12.3.267.

Järvelä, S., Kirschner, P. A., Panadero, E., Malmberg, J., Phielix, C., Jaspers, J., ... \& Järvenoja, H. (2015). Enhancing socially shared regulation in collaborative learning groups: designing for CSCL regulation tools. Educational Technology Research and Development, 63(1), 125-142. https://doi.org/10.1007/s11423-0149358-1

Järvelä, S., Kirschner, P. A., Hadwin, A., Järvenoja, H., Malmberg, J., Miller, M., \& Laru, J. (2016). Socially shared regulation of learning in CSCL: Understanding and prompting individual- and group-level shared regulatory activities. International Journal of Computer-Supported Collaborative Learning, 11(3), 263-280. https://doi.org/10.1007/s11412-016-9238-2.

Järvelä, S., Järvenoja, H., Malmberg, J., Isohätälä, J., \& Sobocinski, M. (2016a). How do types of interaction and phases of self-regulated learning set a stage for collaborative engagement? Learning and Instruction, 43, 3951. https://doi.org/10.1016/j.learninstruc.2016.01.005.

Järvelä, S., Malmberg, J., \& Koivuniemi, M. (2016b). Recognizing socially shared regulation by using the temporal sequences of online chat and logs in CSCL. Learning and Instruction, 42, 1-11. https://doi. org/10.1016/j.learninstruc.2015.10.006.

Järvelä, S., Hadwin, A.F. Malmberg, J. \& Miller. M. (2018). Contemporary perspectives of regulated learning in collaboration. In F. Fischer, C.E. Hmelo-Silver, Reimann, P. \& S. R. Goldman (Eds.). International Handbook of the Learning Sciences (pp. 127-136). New York, NY: Routledge. Taylor \& Francis.

Järvenoja, H., Volet, S., \& Järvelä, S. (2013). Regulation of emotions in socially challenging learning situations: an instrument to measure the adaptive and social nature of the regulation process. Educational Psychology, 33(1), 31-58. https://doi.org/10.1080/01443410.2012.742334.

Järvenoja, H., Järvelä, S., \& Malmberg, J. (2015). Understanding regulated learning in situative and contextual frameworks. Educational Psychologist, 50(3), 204-219. https://doi.org/10.1080/00461520.2015.1075400.

Järvenoja, H., Järvelä, S., \& Malmberg, J. (2017). Supporting groups' emotion and motivation regulation during collaborative learning. Learning and Instruction. https://doi.org/10.1016/j.learninstruc.2017.11.004.

Järvenoja, H., Järvelä, S., Törmänen, T., Näykki, P., Malmberg, J., Mykkänen, A., \& Isohätälä, J. (2018). Capturing motivation and emotion regulation during a learning process. Frontline Learning Research, 6(3), 85-104. https://doi.org/10.14786/flr.v6i3.369.

Johnson, A. M., Azevedo, R., \& D’Mello, S. K. (2011). The temporal and dynamic nature of self-regulatory processes during independent and externally assisted hypermedia learning. Cognition and Instruction, 29(4), 471-504. https://doi.org/10.1080/07370008.2011.610244.

Kimmel, K., \& Volet, S. (2010). Significance of context in university students'(meta) cognitions related to group work: A multi-layered, multi-dimensional and cultural approach. Learning and Instruction, 20(6), 449-464. https://doi.org/10.1016/j.learninstruc.2009.05.004.

Kirschner, P. A., Sweller, J., Kirschner, F., Zambrano, R., \& J. (2018). From Cognitive Load Theory to Collaborative Cognitive Load Theory. International Journal of Computer-Supported Collaborative Learning, 13(2), 213-233. https://doi.org/10.1007/s11412-018-9277-y.

Knight, S., Friend Wise, A., \& Chen, B. (2017). Time for Change: Why Learning Analytics Needs Temporal Analysis. Journal of Learning Analytics, 4(3), 7-17. https://doi.org/10.18608/jla.2017.43.2.

Koivuniemi, M., Panadero, E., Malmberg, J., \& Järvelä, S. (2017). Higher education students' learning challenges and regulatory skills in different learning situations. Infancia y Aprendizaje, 1-37. https://doi. org/10.1080/02103702.2016.1272874

Lajoie, S. P., Lee, L., Poitras, E., Bassiri, M., Kazemitabar, M., Cruz-Panesso, I., ... Lu, J. (2015). The role of regulation in medical student learning in small groups: Regulating oneself and others' learning and emotions. Computers in Human Behavior, 52, 601-616. https://doi.org/10.1016/j.chb.2014.11.073 
Luckin, R. (2018). Machine Learning and Human Intelligence: The Future of Education for the 21st Century. UCL Institute of Education Press, 2018.

Lund, K., \& Suthers, D. (2018). Multivocal analysis. Multiple perspective in analyzing interaction. In F. Fischer, C. E. Hmelo-Silver, S. R. Goldman, \& P. Reimann (Eds.), International Handbook of Learning Sciences (pp. 455-464). New York: Routledge.

Malmberg, J., Järvenoja, H., \& Järvelä, S. (2010). Tracing elementary school students' study tactic use in gStudy by examining a strategic and self-regulated learning. Computers in Human Behavior, 26(5), 1034-1042. https://doi.org/10.1016/j.chb.2010.03.004.

Malmberg, J., Järvenoja, H., \& Järvelä, S. (2013). Patterns in elementary school students' strategic actions in varying learning situations. Instructional Science, 41(5), 933-954. https://doi.org/10.1007/s11251-0129262-1.

Malmberg, J., Järvelä, S., \& Kirschner, P. A. (2014). Elementary school students' strategic learning: does tasktype matter? Metacognition and Learning, 9(2), 113-136. https://doi.org/10.1007/s11409-013-9108-5.

Malmberg, J., Järvelä, S., Järvenoja, H., \& Panadero, E. (2015). Promoting socially shared regulation of learning in CSCL: Progress of socially shared regulation among high-and low-performing groups. Computers in Human Behavior, 52, 562-572. https://doi.org/10.1016/j.chb.2015.03.082.

Malmberg, J., Järvelä, S., \& Järvenoja, H. (2017). Capturing temporal and sequential patterns of self-, co- and socially shared regulation in the context of collaborative learning. Contemporary Journal of Educational Psychology, 49, 160-174. https://doi.org/10.1016/j.cedpsych.2017.01.009.

McCardle, L., \& Hadwin, A. F. (2015). Using multiple, contextualized data sources to measure learners' perceptions of their self-regulated learning. Metacognition and Learning, 10(1), 43-75. https://doi. org/10.1007/s11409-014-9132-0.

Miller, M., \& Hadwin, A. (2015). Scripting and awareness tools for regulating collaborative learning: Changing the landscape of support in CSCL. Computers in Human Behavior, 52, 573-588. https://doi.org/10.1016/j. chb.2015.01.050.

Miyake, N., \& Kirschner, P. A. (2014). The social and interactive dimensions of collaborative learning. In R. K. Sawyer (Ed.), The Cambridge Handbook of the Learning Sciences (2nd ed., pp. 418-438). Cambridge: Cambridge University Press. https://doi.org/10.1017/CBO9781139519526.026.

Molenaar, I., \& Chiu, M. (2014). Dissecting sequences of regulation and cognition: Statistical discourse analysis of primary school children's collaborative learning. Metacognition and Learning, 9(2), 137-160. https://doi. org/10.1007/s11409-013-9105-8.

Molenaar, I., \& Järvelä, S. (2014). Sequential and temporal characteristics of self and socially regulated learning. Metacognition and Learning, 9(2), 75-85. https://doi.org/10.1007/s11409-014-9114-2.

Narciss, S., Proske, A., \& Koerndle, H. (2007). Promoting self-regulated learning in web-based learning environments. Computers in Human Behavior, 23(3), 1126-1144. https://doi.org/10.1016/j. chb.2006.10.006.

Nesbit, J. C., Winne, P. H., Jamieson-Noel, D., Code, J., Zhou, M., Allister, K. M., et al. (2006). Using Cognitive Tools in Gstudy to Investigate How Study Activities Covary with Achievement Goals. Journal of Educational Computing Research, 35(4), 339-358. https://doi.org/10.2190/h3w18321-1260-1443.

Ochoa, X. (2017). Multimodal learning analytics. In Lang, C., Siemens, G., Wise, A., Dragan, G. (Eds). Handbook of learning analytics (pp. 129-141). SOLAR, Society for Learning Analytics and Research.

Paans, C., Molenaar, I., Segers, P.C.J. \& Verhoeven, L.T.W. (2018). Temporal variation in children's selfregulated hypermedia learning. Computers in Human Behavior. In Press. https://doi.org/10.1016/j. chb.2018.04.002

Perry, N. E., Thauberger, C., \& Hutchinson, L. (2010). gStudy traces of children's self-regulated learning in the Lifecycles Learning Kit. Psychological Test and Assessment Modeling, 52(4), 432.

Pijeira-Díaz, H. J., Drachsler, H., Järvelä, S., \& Kirschner, P. A. (2016). Investigating collaborative learning success with physiological coupling indices based on electrodermal activity. In Proceedings of the sixth international conference on learning analytics \& knowledge (pp. 6473). ACM. https://doi.org/10.1145/2883851.2883897

Pijeira-Díaz, H. J., Drachsler, H., Järvelä, S., \& Kirschner, P. A. (2019). Sympathetic arousal commonalities and arousal contagion during collaborative learning: How attuned are triad members? Computers in Human Behavior, 92, 188-197. https://doi.org/10.1016/j.chb.2018.11.008.

Pintrich, P. R. (1995). Understanding self-regulated learning. New Directions for Teaching and Learning, 1995(63), 3-12. https://doi.org/10.1002/tl.37219956304.

Puntambekar, S., \& Stylianou, A. (2005). Designing navigation support in hypertext systems based on navigation patterns. Instructional Science, 33(5-6), 451-481. https://doi.org/10.1007/s11251-005-1276-5. 
Reimann, P., Markauskaite, L., \& Bannert, M. (2014). e-Research and learning theory: What do sequence and process mining methods contribute? British Journal of Educational Technology, 45(3), 528-540. https://doi. org/10.1111/bjet.12146.

Rogat, T. K., \& Adams-Wiggins, K. R. (2014). Other-regulation in collaborative groups: implications for regulation quality. Instructional Science, 42(6), 879-904. https://doi.org/10.1007/s11251-014-9322-9.

Rogat, T. K., \& Adams-Wiggins, K. R. (2015). Interrelation between regulatory and socioemotional processes within collaborative groups characterized by facilitative and directive other-regulation. Computers in Human Behavior, 52, 589-600. https://doi.org/10.1016/j.chb.2015.01.026.

Salmela-Aro, K., Read S., Minkkinen, J., Kinnunen, J. \& Rimpelä, A. (2017). Immigrant status, gender, and school burnout in Finnish lower secondary school students. A longitudinal study. International Journal of Behavioral Development, 42, 2(225-236). https://doi.org/10.1177 $/ 0165025417690264$

Schnaubert, L., \& Bodemer, D. (2017). Prompting and visualising monitoring outcomes: Guiding self-regulatory processes with confidence judgments. Learning and Instruction, 49, 251-262. https://doi.org/10.1016/j. learninstruc.2017.03.004.

Schoor, C., Narciss, S., \& Körndle, H. (2015). Regulation During Cooperative and Collaborative Learning: A Theory-Based Review of Terms and Concepts. Educational Psychologist, 50(2), 97-119. https://oi. org/10.1080/00461520.2015.1038540.

Schunk, D. H., \& Greene, J. A. (2018). Historical, contemporary, and future perspectives on selfregulated learning and performance. In D. Schunk \& J. Greene (Eds.), Handbook of SelfRegulation of Learning and Performance (2nd ed., pp. 1-15). London: Routledge.

Shapiro, A., \& Niederhauser, D. (2004). Learning from hypertext: Research issues and findings. In D. H. Jonassen (Ed.), Handbook of Research on Educational Communications and Technology (pp. 605-520). Taylor \& Francis.

Sobocinski, M., Malmberg, J., \& Järvelä, S. (2017). Exploring temporal sequences of regulatory phases and associated interactions in low-and high-challenge collaborative learning sessions. Metacognition and Learning, 12(2), 275-294. https://doi.org/10.1007/s11409-016-9167-5.

Summers, M., \& Volet, S. (2010). Group work does not necessarily equal collaborative learning: evidence from observations and self-reports. European Journal of Psychology of Education, 25(4), 473-492. https://oi. org/10.1007/s10212-010-0026-5.

Ucan, S., \& Webb, M. (2015). Social Regulation of Learning During Collaborative Inquiry Learning in Science: How does it emerge and what are its functions? International Journal of Science Education, 37(15), 2503-2532. https://doi.org/10.1080/09500693.2015.1083634.

Volet, S. E., \& Järvelä, S. E. (2001). Motivation in learning contexts: Theoretical advances and methodological implications. Amsterdam: Pergamon Press.

Volet, S., \& Vauras, M. (2013). The Study of Interpersonal Regulation in Learning and Its Challenge to the Research Methodology. In S. Volet \& M. Vauras (Eds.), Interpersonal Regulation of Learning and Motivation (pp. 1-13). New York: Routledge.

Webster, E. A., \& Hadwin, A. F. (2014). Emotions and emotion regulation in undergraduate studying: Examining students' reports from a self-regulated learning perspective. Educational Psychology, 35(7), 794-818. https://doi.org/10.1080/01443410.2014.895292.

Winne, P. H. (1982). Minimizing the black box problem to enhance the validity of theories about instructional effects. Instructional Science, 11(1), 13-28. https://doi.org/10.1007/bf00120978.

Winne, P. H. (2015). What is the state of the art in self-, co- and socially shared regulation in CSCL? Computers in Human Behavior, 52, 628-631. https://doi.org/10.1016/j.chb.2015.05.007.

Winne, P. H. (2017). Cognition and metacognition within self-regulated learning. In D. Schunk \& J. Greene (Eds.), Handbook of self-regulation of learning and performance (pp. 52-64). London: Routledge.

Winne, P. H. (2019). Paradigmatic Dimensions of Instrumentation and Analytic Methods in Research on SelfRegulated Learning. Computers in Human Behavior, 96, 285-289.

Winne, P. H., \& Hadwin, A. F. (1998). Studying as self-regulated learning. In D. J. Hacker, J. Dunlosky, \& A. C. Graesser (Eds.), Metacognition in educational theory and practice (pp. 277-304). Mahwah: Lawrence Erlbaum.

Winne, P. H., \& Hadwin, A. F. (2008). The weave of motivation and self-regulated learning. In D. H. Schunk \& B. J. Zimmerman (Eds.), Motivation and Self-Regulated Learning: Theory, Research and Applications (pp. 297-314). New York: Lawrence Erlbaum Associates.

Wise, A. F., \& Chiu, M. M. (2011). Analyzing temporal patterns of knowledge construction in a role-based online discussion. International Journal of Computer-Supported Collaborative Learning, 6(3), 445-470. https://oi.org/10.1007/s11412-011-9120-1. 
Zheng, J., Xing, W., \& Zhu, G. (2019). Examining sequential patterns of self-and socially shared regulation of STEM learning in a CSCL environment. Computers \& Education, 136, 34 48. https://doi.org/10.1016/j. compedu.2019.03.005.

Zimmerman, B. (2013). From cognitive modeling to self-regulation: A Social cognitive career path. Educational Psychologist, 48, 135-147. https://doi.org/10.1080/00461520.2013.794676.

Zschocke, K., Wosnitza, M., \& Bürger, K. (2016). Emotions in group work: insights from an appraisal-oriented perspective. European Journal of Psychology of Education, 31(3), 359-384. https://doi.org/10.1007/s10212015-0278-1.

Publisher's note Springer Nature remains neutral with regard to jurisdictional claims in published maps and institutional affiliations. 